PROCEEDINGS OF THE AMERICAN MATHEMATICAL SOCIETY

Volume 124, Number 10, October 1996

\title{
ON THE DIMENSION OF HILBERT SPACE REMAINDERS
}

\author{
JAN J. DIJKSTRA AND JAN VAN MILL
}

(Communicated by James E. West)

Every space is assumed to be separable and metric. A space is called (strongly) countably dimensional if it can be written as a countable union of (closed) finite dimensional subspaces. A space $X$ is called strongly infinite dimensional if the space admits an essential system $\left(F_{n}, G_{n}\right)_{n=1}^{\infty}$, i.e. $F_{n}$ and $G_{n}$ are disjoint closed subsets of $X$ such that if $S_{n}$ is a closed separator of $F_{n}$ and $G_{n}$ for each $n$, then $\bigcap_{n=1}^{\infty} S_{n}$ is nonempty. The sequence of left and right endfaces of the Hilbert cube is the standard example of an essential system.

A well-known theorem of Engelking [E] states that every autohomeomorphism $h$ of an $n$-dimensional space $X$ can be extended to a homeomorphism $\tilde{h}: C \rightarrow C$, where $C$ is an $n$-dimensional compactification of $X$ (and hence we have a $\leq n$ dimensional remainder). We consider the question of whether similar results can be obtained for infinite dimensional spaces, i.e. is it possible to put a bound on the dimension of the remainder? The following example shows that the answer is no if we allow incomplete spaces. Consider the Hilbert cube $Q=[0,1]^{\mathbf{N}}$ and the strongly countably dimensional pseudoboundary $\sigma=\left\{x \in Q: x_{i}=0\right.$ from some index on $\}$. It was shown by R. D. Anderson that $Q \backslash \sigma$ is homeomorphic to Hilbert space (see [BP, Theorem V.5.1]). The following proposition is a slight improvement of the known result that the remainder of every compactification of $\sigma$ contains a copy of $Q$.

Proposition 1. The remainder of every completion of $\sigma$ contains a dense copy of Hilbert space.

Proof. Let $C$ be a completion of $\sigma$. According to [La] there exist a $G_{\delta}$-set $A$ in $C$, a $G_{\delta}$-set $B$ in $Q$, and a homeomorphism $h: A \rightarrow B$ such that $\sigma \subset A, \sigma \subset B$, and $h \mid \sigma$ is the identity. Since $Q \backslash B$ is $\sigma$-compact, it is negligible in the Hilbert space $Q \backslash \sigma$ (see [A]). So $B \backslash \sigma$ and $A \backslash \sigma$ are Hilbert spaces.

We turn to complete spaces. According to [Le] every complete space can be compactified by adding a strongly countably dimensional remainder. This fact also follows from the aforementioned result that Hilbert space can be compactified to a Hilbert cube by using $\sigma$ as remainder. So the question naturally arises of whether every autohomeomorphism of a complete space can be "compactified" by adding a strongly countably dimensional remainder. Let us have a closer look at Hilbert

Received by the editors November 19, 1995 and, in revised form, November 27, 1995.

1991 Mathematics Subject Classification. Primary 54F45, 57N20.

The first author is pleased to thank the Vrije Universiteit in Amsterdam for its hospitality and support. 
space which we now represent by $s=\mathbf{R}^{\mathbf{Z}}=\prod_{i=-\infty}^{\infty} \mathbf{R}$. Let $\alpha$ stand for the "left shift" on $s$, i.e. $\alpha(x)_{i}=x_{i+1}$ for $i \in \mathbf{Z}$.

Proposition 2. If $\alpha$ extends over a compactification to a continuous $\tilde{\alpha}: C \rightarrow C$, then $C \backslash s$ contains strongly infinite dimensional continua.

Proof. Let $\left\{A_{1}, A_{2}, \ldots\right\}$ be a partition of $\mathbf{N}$ into infinitely many infinite subsets. We define the following sequence of disjoint pairs of closed subsets of $s$ : for $n \in \mathbf{N}$ and $\varepsilon \in\{0,1\}$,

$$
F_{n}^{\varepsilon}=\left\{\left(x_{i}\right) \in s: x_{i}=\varepsilon \text { if for some } k \in A_{n} \text { we have } k^{2} \leq i<(k+1)^{2}\right\} .
$$

Let $\tilde{F}_{n}^{\varepsilon}$ be the closure in $C$ of $F_{n}^{\varepsilon}$. We first show that $\tilde{F}_{n}^{0}$ and $\tilde{F}_{n}^{1}$ are disjoint. Let $U_{0}$ and $U_{1}$ be two disjoint closed neighbourhoods of $(\ldots, 0,0,0, \ldots)$ and $(\ldots, 1,1,1, \ldots)$ in $C$. Then there is an $N \in \mathbf{N}$ such that $\bigcap_{i=-N}^{N} \pi_{i}^{-1}(0) \subset U_{0}$ and $\bigcap_{i=-N}^{N} \pi_{i}^{-1}(1) \subset$ $U_{1}$, where $\pi_{i}: s \rightarrow \mathbf{R}$ stands for the projection on the $i$ th coordinate. Select a $k \in A_{n}$ such that $k \geq N$. Put $m=k^{2}+k$. If $x \in F_{n}^{\varepsilon}$, then $x_{i}=\varepsilon$ for $k^{2} \leq i \leq k^{2}+2 k$. Since $\alpha^{m}$ is a shift to the left over $k^{2}+k$ positions we have $\alpha^{m}(x)_{i}=\varepsilon$ for $-k \leq i \leq k$. So $\alpha^{m}\left(F_{n}^{0}\right) \subset U_{0}$ and $\alpha^{m}\left(F_{n}^{1}\right) \subset U_{1}$ and since $U_{0}$ and $U_{1}$ are compact and disjoint we have that $\tilde{\alpha}^{n}\left(\tilde{F}_{n}^{0}\right)$ and $\tilde{\alpha}^{n}\left(\tilde{F}_{n}^{1}\right)$ are disjoint. Hence $\tilde{F}_{n}^{0}$ and $\tilde{F}_{n}^{1}$ are disjoint.

We define the imbedding $\beta$ of the space $X=[0, \infty) \times Q$ into $s$ as follows: for $a \geq 0, x=\left(x_{j}\right) \in Q$, and $i \in \mathbf{Z}$,

$$
\beta(a, x)_{i}= \begin{cases}a, & \text { if } i \leq 0 \\ x_{j}, & \text { if } k^{2} \leq i<(k+1)^{2} \text { for some } k \text { and } j \text { with } k \in A_{j} .\end{cases}
$$

Observe that $\beta$ is a closed imbedding of a locally compact space in $s$ and hence $K=\operatorname{cl}_{C}(\beta(X)) \backslash \beta(X)$ is a compactum in $C \backslash s$. Since $K=\bigcap_{i=1}^{\infty} \operatorname{cl}_{C}(\beta([i, \infty) \times Q))$, it is a continuum. Let $\beta_{a}: Q \rightarrow s$ be defined by $\beta_{a}(x)=\beta(a, x)$ for $(a, x) \in X$.

Now we prove that $K$ is strongly infinite dimensional. Assume that $S_{n}$ is a closed separator in $K$ of $\tilde{F}_{n}^{0} \cap K$ and $\tilde{F}_{n}^{1} \cap K$. Since $K$ is compact, we can find for each $n$ a closed separator $\tilde{S}_{n}$ of $\tilde{F}_{n}^{0}$ and $\tilde{F}_{n}^{1}$ in $C$ such that $\tilde{S}_{n} \cap K=S_{n}$. Put $\tilde{S}_{\infty}=\bigcap_{n=1}^{\infty} \tilde{S}_{n}$. Observe that for each $a \geq 0$ the sets $\beta_{a}^{-1}\left(F_{n}^{0}\right)$ and $\beta_{a}^{-1}\left(F_{n}^{1}\right)$ are precisely the $n$ endfaces of the Hilbert cube $Q$ and hence they form an essential system for $n \in \mathbf{N}$. So we may conclude that $\bigcap_{n=1}^{\infty} \beta_{a}^{-1}\left(\tilde{S}_{n}\right)$ and hence $\beta_{a}(Q) \cap \tilde{S}_{\infty}$ are nonempty. Since $\pi_{0}(\beta(a, x))=a$ we have $\pi_{0}\left(\beta(X) \cap \tilde{S}_{\infty}\right)=[0, \infty)$. So $\beta(X) \cap \tilde{S}_{\infty}$ is not compact. Since $\operatorname{cl}_{C}(\beta(X)) \cap \tilde{S}_{\infty}$ is compact, we may conclude that $\bigcap_{n=1}^{\infty} S_{n}=K \cap \tilde{S}_{\infty}$ is nonempty.

Propositions 1 and 2 suggest the following questions. If $\alpha$ extends over a compactification to a homeomorphism $\tilde{\alpha}: C \rightarrow C$, does $C \backslash s$ contain a Hilbert cube? And if $h$ is an autohomeomorphism of a (strongly) countably dimensional complete space $X$, can $h$ be extended to a homeomorphism $\tilde{h}: C \rightarrow C$, where $C$ is a compactification of $X$ with (strongly) countably dimensional remainder?

\section{REFERENCES}

[A] R. D. Anderson, Strongly negligible sets in Fréchet manifolds, Bull. Amer. Math. Soc. 75 (1969), 64-67. MR 38:6634

[BP] C. Bessaga and A. Pełczyński, Selected Topics in Infinite-Dimensional Topology, PWN, Warszawa, 1975. MR 57:17657 
[E] R. Engelking, Sur la compactification des espaces métriques, Fund. Math. 48 (1960), 321324. MR 23:A1347

[La] M. Lavrentiev, Contributions à la théorie des ensembles homéomorphes, Fund. Math. 6 (1924), 149-160.

[Le] A. Lelek, On the dimension of remainders in compact extensions, Soviet Math. Dokl. 6 (1965), 136-140

Department of Mathematics, The University of Alabama, Box 870350, Tuscaloosa, Alabama 35487-0350

E-mail address: jdijkstr@ua1vm.ua.edu

Faculteit Wiskunde en Informatica, Vrije Universiteit, De Boelelaan 1081A, 1081 HV Amsterdam, The Netherlands

E-mail address: vanmill@cs.vu.nl 